\title{
Knockdown of STEAP4 inhibits insulin-stimulated glucose transport and GLUT4 translocation via attenuated phosphorylation of Akt, independent of the effects of EEA1
}

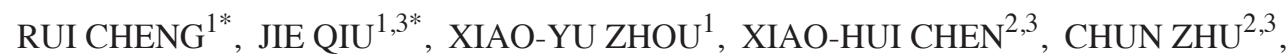 \\ DA-NI QIN ${ }^{2,3}, \mathrm{JI}_{-\mathrm{WU}} \mathrm{WANG}^{4}$, YU-HUI NI ${ }^{4}, \mathrm{CHEN}^{-B O} \mathrm{JI}^{3}$ and XI-RONG GUO ${ }^{2,3}$ \\ ${ }^{1}$ Department of Newborn Infants, Nanjing Children's Hospital of Nanjing Medical University, Nanjing 210008; \\ ${ }^{2}$ Department of Pediatrics, Nanjing Maternity and Child Health Hospital of Nanjing Medical University, \\ Nanjing 210004; ${ }^{3}$ Institute of Pediatrics of Nanjing Medical University, Nanjing 210029, P.R. China; \\ ${ }^{4}$ Allele Biotechnology and Pharmaceuticals Inc., San Diego, CA 92121, USA
}

Received October 25, 2010; Accepted February 11, 2011

DOI: $10.3892 / \mathrm{mmr} .2011 .443$

\begin{abstract}
The aim of this study was to investigate whether the early endosome antigen 1 (EEA1) and/or PI3K pathway is involved in the molecular mechanisms underlying the effects of the six-transmembrane protein of prostate 4 (STEAP4; also called STAMP2 and TIARP) on the insulin sensitivity of human adipocytes. Our data demonstrated that siRNA-mediated STEAP4 deficiency significantly decreased insulin-stimulated glucose transport in mature human adipocytes by decreasing GLUT4 translocation to the plasma membrane through attenuated Akt phosphorylation. We further found that EEA1 may not be involved in the mechanisms underlying the effects of STEAP4 on insulin-stimulated glucose uptake and GLUT4 translocation, as indicated by the results that i) STEAP4 does not alter the effects of EEA1 on insulin-stimulated glucose uptake and GLUT4 translocation; ii) STEAP4 does not modify the expression of EEA1 protein; and iii) STEAP4 does not interact with EEA1 according to FRET analysis. In conclusion, this study revealed that the knockdown of STEAP4 inhibits insulin-stimulated glucose transport and GLUT4 translocation via the attenuated phosphorylation of Akt, independent of the effects of EEA1.
\end{abstract}

Correspondence to: Professor Xi-Rong Guo, Department of Pediatrics, Nanjing Maternity and Child Health Hospital of Nanjing Medical University, 123 Mochou Road, Jiangsu, Nanjing 210004, P.R. China

E-mail:xrguo@njmu.edu.cn

Dr Chen-Bo Ji, Institute of Pediatrics of Nanjing Medical University, Nanjing 210029, P.R. China

E-mail: chenboji@njmu.edu.cn

*Contributed equally

Key words: six-transmembrane protein of prostate 4, early endosome antigen 1, Akt, glucose transport, GLUT4 translocation

\section{Introduction}

Insulin resistance is a common feature of obesity and is implicated in its metabolic complications, including type 2 diabetes and cardiovascular disease. Various pathways and factors in white adipose tissue have been proposed to contribute to obesity-associated insulin resistance (1). Despite years of investigation, the molecular mechanisms that link obesity and insulin resistance have not been completely elucidated, and remain the subject of intensive research.

Our previous studies showed that the six-transmembrane protein of prostate 4 (STEAP4; also called STAMP2 and TIARP), a novel obesity-related gene abundantly expressed in human omental adipose tissue, participates in the regulation of insulin in human adipocytes (2-4). Other studies on STEAP4 have also revealed that this gene is associated with insulin resistance $(5,6)$. However, the molecular events underlying the effects of STEAP4 on the regulation of insulin sensitivity have yet to be fully elucidated.

Korkmaz et al demonstrated that STEAP4 localizes to vesicular-tubular structures in the cytosol and colocalizes with the early endosome antigen 1 (EEA1), which has been proposed to mediate early endosome fusion (7). EEA1 was identified to interact with PI3P, a product of PI3K, via a double zinc finger FYVE domain at its C terminus (8-10). Treating cells with the PI3K inhibitor wortmannin causes the dissociation of EEA1 from early endosomes (11). At present, it is generally well accepted that PI3K is required for fusing GLUT4-containing vesicles with the plasma membrane, while insulin-stimulated glucose uptake is mediated primarily by the rapid movement of GLUT4 from a latent intracellular compartment to the cell surface (12). Therefore, we speculated that the involvement of the EEA1 and/or PI3K pathway in the molecular mechanisms underlying the effects of STEAP4 may influence the insulin sensitivity of human adipocytes.

\section{Materials and methods}

Plasmids. The STEAP4 and EEA1 RNAi targeting sequences were designed using the RNAi design algorithm SVM RNAi 
2.0 (Chang Bioscience Inc., Castro Valley, CA, USA). Four predicted target sequences for each were synthesized and cloned into the pre-cut retroviral vector pCHAC-shRNAHygro according to the manufacturer's instructions (Allele Biotech and Orbigen Inc., San Diego, CA, USA). The specific sequence for green fluorescent protein (GFP) was used as a negative control for the RNAi experiment (Allele Biotech and Orbigen Inc., San Diego, CA, USA).

To overexpress STEAP4, the open reading frame (ORF) of STEAP4 was amplified using the primers 5'-CGC GCT CGA GGC CAC CAT GGA GAA AAC TTG TAT AGA-3' and 5'-CGA AGG CGG CCG CCT AGT GTT TTG AGT TCC TTT CC-3' and inserted into the XhoI/NotI sites of the retroviral pCHAC-MCS-IRES-NeoR vector (Allele Biotech and Orbigen Inc.).

The GLUT4-7Myc-GFP reporter was a kind gift from Jonathan S. Bogan (13). For easy packaging, the GLUT47Myc-GFP expression cassette was sub-cloned into the BamHI/SalI sites of the retroviral pCHAC-MCS-IRES-NeoR vector (Allele Biotech and Orbigen Inc.).

Virus generation and infection. Ampho packaging cells (Allele Biotech and Orbigen Inc.) were transfected with retroviral expression vectors using GenePORTER 2 reagent (Genlantis Inc., San Diego, CA, USA) following the manufacturer's instructions. Supernatants were collected $48 \mathrm{~h}$ after transfection, filtered through a $0.45-\mu \mathrm{m}$ membrane, supplemented with hexadimethrine bromide (polybrene) to a final concentration of $4 \mu \mathrm{g} / \mathrm{ml}$, and applied directly to the human preadipocytes.

Cell culture and differentiation of human preadipocytes. Human preadipocytes (ScienCell Research Laboratories, San Diego, CA, USA) were maintained in Dulbecco's modified Eagle's medium (DMEM; Gibco, Carlsbad, CA, USA) containing $10 \%$ fetal bovine serum (FBS; Gibco, Carlsbad, CA, USA), $100 \mathrm{U} / \mathrm{ml}$ penicillin and $50 \mu \mathrm{g} / \mathrm{ml}$ streptomycin at $37^{\circ} \mathrm{C}$ in $5 \% \mathrm{CO}_{2}$. After infection, confluent human preadipocytes (day 0) were cultured in serum-free DMEM containing $50 \mathrm{nM}$ insulin, $100 \mathrm{nM}$ dexamethasone, $0.5 \mathrm{mM}$ 3-isobutyl1-methylxanthine, and $100 \mu \mathrm{M}$ rosiglitazone. The medium was changed every 2 days for the first 4 days. Thereafter, the medium was replaced by serum-free DMEM containing insulin $(50 \mathrm{nM})$, and this was changed every 2 days until lipid droplets had accumulated (days 14-17).

Glucose uptake. 2-[N-(7-nitrobenz-2-oxa-1, 3-diazol-4-yl) amino]-2-deoxy-D-glucose (2-NBDG) is a fluorescent D-glucose derivative for monitoring glucose uptake into single, living mammalian cells $(14,15)$. Before the cells were assayed, they were starved in DMEM without fetal bovine serum for at least $3 \mathrm{~h}$. After being washed with Krebs-Ringer phosphate buffer (KRB) containing $2.8 \mathrm{mM}$ glucose, the cells were incubated in $500 \mu \mathrm{l} \mathrm{KRB}$ for $30 \mathrm{~min}$ at $37^{\circ} \mathrm{C}$. They were then stimulated with/without $100 \mathrm{nM}$ insulin for $30 \mathrm{~min}$ in KRB at $37^{\circ} \mathrm{C}$. Uptake was initiated by the addition of a fluorescent derivative of 2-NBDG (Molecular probe) at $600 \mu \mathrm{mol} / 1$ in $2.8 \mathrm{mmol} / \mathrm{l}$ glucose containing KRB. The reaction was terminated by quickly washing the cells with ice-cold KRB after $15 \mathrm{~min}$. The fluorescence of 2-NBDG was measured by the Cytofluor Fluorescence Multi-Well Plate Reader Series 4000 (Applied Biosystems, Foster City, CA, USA).

GLUT4 translocation. Cells infected with GLUT4-7Myc-GFP were starved in DMEM without fetal bovine serum for at least $3 \mathrm{~h}$ before insulin stimulation. After treatment in the presence or absence of insulin $(100 \mathrm{nM})$ for $30 \mathrm{~min}$, the cells were quickly transferred and washed with cold phosphate-buffered saline (PBS) at $4^{\circ} \mathrm{C}$. Cells were incubated with a 1:500 dilution of anti-Myc (9E10) (abcam) as cites for $1.5 \mathrm{~h}$ and then washed twice in PBS for 5 min each time. After incubation with a 1:200 dilution of PE-conjugated $\mathrm{F}\left(\mathrm{ab}^{\prime}\right) 2$ donkey anti-mouse IgG for $45 \mathrm{~min}$, the cells were washed three times in PBS for 10 min each time. GLUT4 translocation was measured using the CytoFluor Fluorescence Multi-Well Plate Reader Series 4000 (Applied Biosystems) $(13,16)$.

Western blotting. Total or phosphorylated protein was extracted as previously described (17). The protein was quantified using the bicinchonic acid (BCA) protein assay kit (Pierce, Rockford, IL, USA) in accordance with the manufacturer's instructions. After sodium dodecyl sulfate-polyacrylamide gel electrophoresis (SDS-PAGE), the proteins $(20 \mu \mathrm{g} / \mathrm{lane})$ were electrophoretically transferred onto a nitrocellulose membrane (Whatman, London, UK). The membrane was blocked with Tris-buffered saline containing Tween-20 (TBST; $0.14 \mathrm{~mol} / \mathrm{l}$ $\mathrm{NaCl}, 0.02 \mathrm{~mol} / \mathrm{l}$ Tris base, $\mathrm{pH}$ 7.6, and 0.1\% Tween) and 3\% bovine serum albumin (BSA) for $1 \mathrm{~h}$ at room temperature, then hybridized with primary antibodies at an appropriate dilution at $4^{\circ} \mathrm{C}$ overnight. The membrane was then washed five times with TBST for 5 min each time, after which it was incubated with horseradish peroxidase-conjugated secondary antibodies for $1 \mathrm{~h}$ at room temperature, washed with TBST and developed with the enhanced chemiluminescence (ECL) kit (Amersham Biosciences, Piscataway, NJ, USA).

Fluorescence resonance energy transfer. STEAP4 were inserted in the mTFP1-N vector (Allele Biotech and Orbigen Inc.). EEA1 cDNA (MGC: 190721) was purchased from Open Biosystems (Huntsville, AL, USA) and cloned into the BamHI/ SalI sites in the vector YFP (Clontech, Palo Alto, CA, USA). Positive control mTFP1-YFP linked with SGLRSPPVAT was a kind gift from Allele Biotech. HEK293 cells were transiently co-transfected with a total of $1 \mu \mathrm{g}$ of mTFP1-tagged STEAP4 and YFP-tagged EEA1 or the mTFP1/YFP negative control vectors at a 1:1 ratio or with $1 \mu \mathrm{g}$ positive control mTFP1-YFP. After $24 \mathrm{~h}$, the cells were washed twice in PBS at room temperature and then fixed with $4 \%$ paraformaldehyde for $10 \mathrm{~min}$. Subsequently, paraformaldehyde was removed and the cells were washed three times with PBS. The emission fluorescence of mTFP1 and YFP was immediately measured using a Mithras LB940 reader (Berthold Technologies, Bad Herrenalb, Germany) equipped with a $430 \mathrm{~nm}$ excitation filter and a filter wheel with $480 \mathrm{~nm}$ (CFP) and $530 \mathrm{~nm}$ (YFP) emission filters.

Statistical analysis. Each experiment was performed at least three times. Values are presented as the mean \pm SD. Statistical analyses were performed using the Student's t-test, f-test or q-test. The statistical significance was defined as $\mathrm{P}<0.05$. 
A

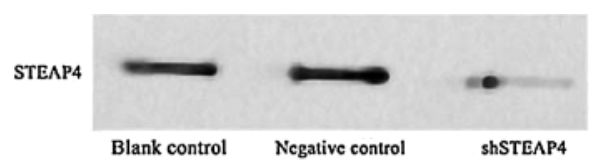

B

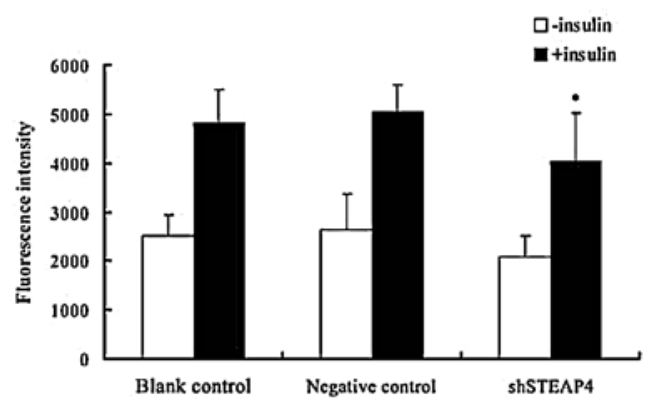

C

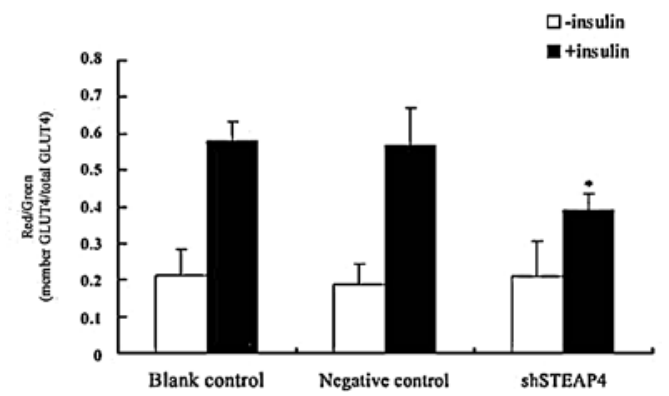

Figure 1. Effects of STEAP4 on basal and insulin-stimulated glucose uptake and GLUT4 translocation in human adipocytes. STEAP4 shRNA retrovirus was prepared and used to infect human preadipocytes. The expression of STEAP4 was analyzed by Western blotting after cells differentiated into mature adipocytes. Cells infected with the STEAP4 shRNA virus showed a marked decrease in STEAP4 (A). After serum starvation for $3 \mathrm{~h}$, the cells were incubated with (black columns) or without (white columns) insulin $(100 \mathrm{nM})$ for $30 \mathrm{~min}$, followed by measurement of glucose uptake (B) and GLUT4 translocation $(\mathrm{C}) .{ }^{*} \mathrm{P}<0.05$.

\section{Results}

Effects of STEAP4 on basal and insulin-stimulated glucose uptake and GLUT4 translocation in human adipocytes. To determine the effects of STEAP4 on basal and insulinstimulated glucose uptake and GLUT4 translocation in human adipocytes, a retroviral vector pCHAC-shSTEAP4-Hygro plasmid was generated. The virus was prepared and used to infect the human preadipocytes, and the expression of STEAP4 was analyzed by Western blotting after these cells differentiated into mature adipocytes. As shown in Fig. 1A, infection of cells with shRNA virus (target sequence 5'-GGG CTG AGT TAA GTG AAA G-3') resulted in a marked decrease in STEAP4, whereas other sequences had no effects (data not shown).

In STEAP4-knockdown cells, basal glucose uptake was similar to that observed in the controls; however, insulinstimulated glucose uptake was approximately $20 \%$ lower in STEAP4-knockdown cells than in the control cells (Fig. 1B). In adipocytes, insulin-stimulated glucose uptake is dependent on the translocation of the insulin-responsive glucose transporter GLUT4 from intracellular storage compartments to the plasma membrane. Our data demonstrated that shRNA-mediated

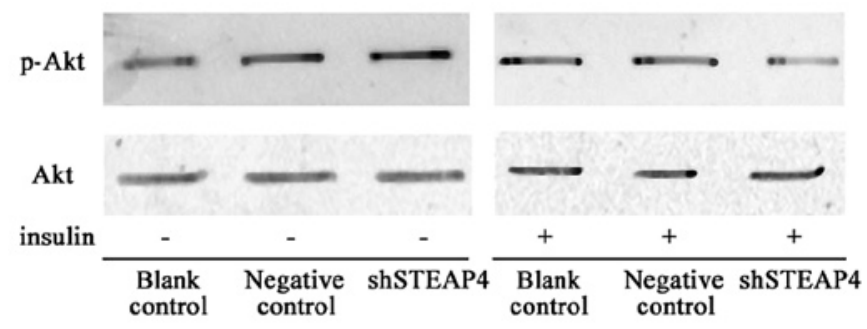

Figure 2. Effects of STEAP4 on protein expression and insulin-stimulated phosphorylation of Akt. Human preadipocytes infected with STEAP4 shRNA retrovirus, negative virus, or uninfected cells were grown and differentiated into mature adipocytes. After incubation with or without insulin $(100 \mathrm{nM})$ for $30 \mathrm{~min}$, the cell lysates were analyzed by SDS-PAGE, blotted onto a membrane, and then probed with antibodies against Akt and the phosphorylated form of this protein.

STEAP4 deficiency significantly decreased insulin-stimulated GLUT4 translocation to the plasma membrane, but did not alter basal GLUT4 translocation (Fig. 1C).

Effects of STEAP4 on protein expression and insulin-stimulated phosphorylation of Akt. Next, the effects of STEAP4 on protein expression and insulin-stimulated phosphorylation of Akt were investigated. The data showed that the RNAimediated depletion of STEAP4 resulted in a significant inhibition of the insulin-induced phosphorylation of Akt, while there was no significant difference in the total protein content of Akt (Fig. 2).

Relationship between STEAP4 and EEA1. The shEEA1 retrovirus was prepared and used to infect human preadipocytes. The expression of EEA1 was analyzed by Western blotting once the cells had differentiated into mature adipocytes. Infection of cells with the shRNA virus (target sequence 5'-GCG GAG TTT AAG CAG CTA C-3') resulted in the depletion of EEA1 (Fig. 3A), whereas other sequences had no effects (data not shown). In mature human adipocytes, shRNA-mediated EEA1 deficiency significantly decreased insulin-stimulated glucose uptake (Fig. 3C) and insulin-stimulated GLUT4 translocation to the plasma membrane (Fig. 3D).

Human mature adipocytes in which EEA1 was stably knocked down and STEAP4 was overexpressed were prepared as described above (Fig. 3B). The data showed that the overexpression of STEAP4 did not alter the effects of EEA1 on insulin-stimulated glucose uptake (Fig. 3C) and GLUT4 translocation (Fig. 3D).

To further demonstrate the relationship between STEAP4 and EEA1, the expression of EEA1 was analyzed in cells infected with the STEAP4 shRNA virus, STEAP4 expression virus, empty vector, and in uninfected cells. As shown in Fig. 3E, the expression of EEA1 was invariant in human mature adipocytes that showed neither stable knockdown nor STEAP4 overexpression.

Fluorescence resonance energy transfer (FRET) has emerged as a powerful tool to determine protein-protein interactions in intact cells. In the present study, FRET was used to elucidate the interaction between EEA1 and STEAP4. The data showed that EEA1 does not interact with STEAP4 (Fig. 3F). 
A

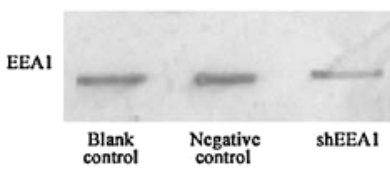

C

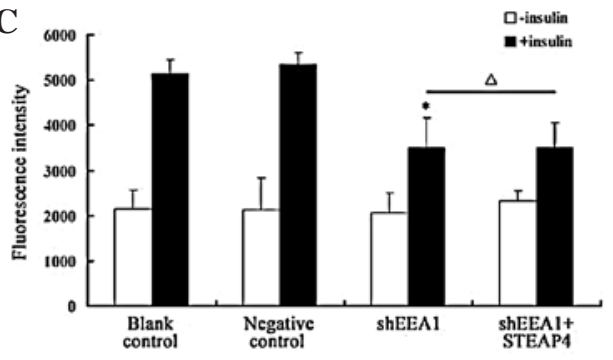

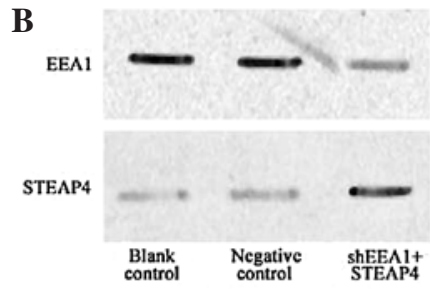

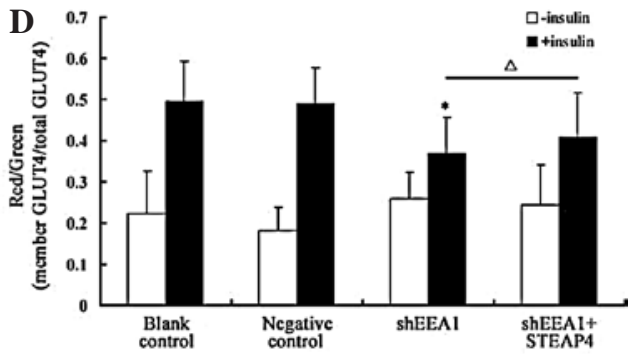

$\mathbf{E}$

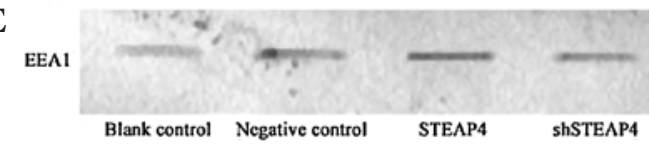

F

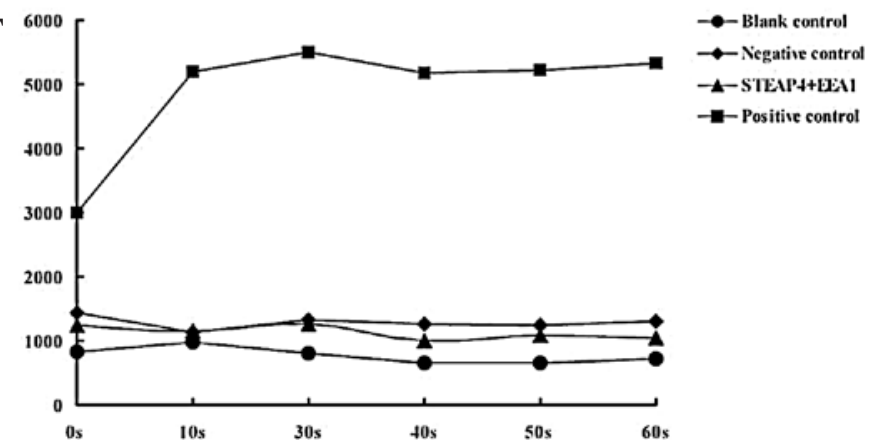

Figure 3. Relationship between STEAP4 and EEA1. Human mature adipocytes with stably knocked down EEA1 (and STEAP4 overexpression) were prepared and validated by Western blotting (A and B). ShRNA-mediated EEA1 deficiency significantly decreased insulin-stimulated glucose uptake (C) and GLUT4 translocation to the plasma membrane (D), while STEAP4 did not influence these effects of EEA1 (C and D). As shown in (E), the expression of EEA1 was invariant in human mature adipocytes that showed neither stable knockdown nor overexpression of STEAP4. Furthermore, FRET data indicated that STEAP4 does not interact with EEA1 (F). "P $<0.05 ;{ }^{\wedge} \mathrm{P}>0.05$.

\section{Discussion}

Adipocytes exhibit considerable increases in the rate of glucose transport in response to insulin by virtue of the expression of a unique glucose transporter, GLUT4 (18-20). GLUT4 is stored in intracellular vesicles and is translocated to the plasma membrane upon insulin stimulation in adipocytes. Our previous data showed that STEAP4 overexpression increases the insulin sensitivity of mature human adipocytes by promoting insulin-stimulated glucose uptake (4). In this study, we further demonstrated that siRNA-mediated STEAP4 deficiency significantly decreased insulin-stimulated glucose transport in mature adipocytes by decreasing GLUT4 translocation to the plasma membrane.

Multiple studies have begun to dissect the molecular machinery involved in the trafficking of GLUT4 vesicles. It is increasingly apparent that insulin-induced GLUT4 translocation in adipocytes requires dynamic actin remodeling at the inner surface of the plasma membrane as well as the perinuclear region (21), and the activation of phosphatidylinositol
(PI3K) regulates the dynamic actin rearrangement required for GLUT4 translocation (22-25). A well-documented case of a PI3K downstream target is the protein Ser/Thr kinase Akt, which is activated through membrane localization (26) and Ser/Thr phosphorylation (27-28). To further investigate the mechanisms by which the RNAi-mediated depletion of STEAP4 decreases insulin-stimulated GLUT4 translocation and glucose uptake, we examined the phosphorylation levels of Akt and found that STEAP4 knockdown attenuated the phosphorylation level of Akt. Therefore, we deduced that RNAi-mediated knockdown of STEAP4 reduced insulinstimulated GLUT4 translocation through attenuated Akt phosphorylation.

Foster et al have demonstrated that internalized GLUT4 travels through the early endosome defined by the presence of EEA1 (29), which is not only a marker of early endosomes but also a functional molecule for the tethering and docking of endosomal vesicles. The FYVE domain, which was originally identified in EEA1, specifically interacts with PI3P (8-11), the major product of PI $3 \mathrm{~K}$ in yeast and mammalian cells $(11,30)$. 
Otherwise, STEAP4 was found to colocalize with EEA1 (7). Therefore, we presumed that EEA1 might be involved in the effects of STEAP4 on glucose transport and GLUT4 translocation, as well as the molecular events underlying these effects. In this study, we found that i) STEAP4 does not alter the effects of EEA1 on insulin-stimulated glucose uptake and GLUT4 translocation; ii) STEAP4 does not modify the expression of EEA1 protein; and iii) STEAP4 does not interact with EEA1 according to FRET analysis. In summary, the data demonstrate that EEA1 may not take part in the mechanisms underlying the effects of STEAP4 on insulin-stimulated glucose uptake and GLUT4 translocation.

In conclusion, this study revealed that the knockdown of STEAP4 inhibits insulin-stimulated glucose transport and GLUT4 translocation via attenuated phosphorylation of Akt, independent of the effects of EEA1.

\section{Acknowledgements}

This study was supported by grants from the National Natural Science Foundation of China (no. 30772364), the Foundation of the Ministry of Education, China (no. 20070312001), the Natural Science Foundation of Jiangsu Province, China (no. BK2007230), and the Graduate Research and Innovation Plan of Jiangsu Province (no. CX09B_256Z).

\section{References}

1. Gustafson B, Hammarstedt A, Andersson CX and Smith U: Inflamed adipose tissue: a culprit underlying the metabolic syndrome and atherosclerosis. Arterioscler Thromb Vasc Biol 27: 2276-2283, 2007.

2. Qiu J, Ni YH, Gong HX, et al: Identification of differentially expressed genes in omental adipose tissues of obese patients by suppression subtractive hybridization. Biochem Biophys Res Commun 352: 469-478, 2007.

3. Zhang CM, Chi X, Wang B, et al: Downregulation of STEAP4, a highly-expressed TNF-alpha-inducible gene in adipose tissue, is associated with obesity in humans. Acta Pharmacol Sin 29: 587-592, 2008.

4. Chen XH, Zhu C, Ji CB, et al: STEAP4, a gene associated with insulin sensitivity, is regulated by several adipokines in human adipocytes. Int J Mol Med 25: 361-367, 2010.

5. Wellen KE, Fucho R, Gregor MF, et al: Coordinated regulation of nutrient and inflammatory responses by STAMP2 is essential for metabolic homeostasis. Cell 129: 537-548, 2007.

6. Arner P, Stenson BM, Dungner E, Näslund E, Hoffstedt J, Ryden $M$ and Dahlman I: Expression of six transmembrane protein of prostate 2 in human adipose tissue associates with adiposity and insulin resistance. J Clin Endocrinol Metab 93 2249-2254, 2008.

7. Korkmaz CG, Korkmaz KS, Kurys P, et al: Molecular cloning and characterization of STAMP2, an androgen-regulated six transmembrane protein that is overexpressed in prostate cancer. Oncogene 24: 4934-4945, 2005.

8. Burd CG and Emr SD: Phosphatidylinositol(3)-phosphate signaling mediated by specific binding to RING FYVE domains. Mol Cell 2: 157-162, 1998.

9. Gaullier JM, Simonsen A, D'Arrigo A, Bremnes B, Stenmark H and Aasland R: FYVE fingers bind PtdIns(3)P. Nature 394 432-433, 1998

10. Patki V, Lawe DC, Corvera S, Virbasius JV and Chawla A: A functional PtdIns(3)P-binding motif. Nature 394: 433-434, 1998.
11. Patki V, Virbasius J, Lane WS, Toh BH, Shpetner HS and Corvera S: Identification of an early endosomal protein regulated by phosphatidylinositol 3-kinase. Proc Natl Acad Sci USA 94: 7326-7330, 1997.

12. Birnbaum MJ: The insulin-sensitive glucose transporter. Int Rev Cytol 137: 239-297, 1992.

13. Bogan JS, McKee AE and Lodish HF: Insulin-responsive compartments containing GLUT4 in 3T3-L1 and CHO cells: regulation by amino acid concentrations. Mol Cell Biol 21: 4785-4806, 2001.

14. Bogan JS, Hendon N, McKee AE, Tsao TS and Lodish HF: Functional cloning of TUG as a regulator of GLUT4 glucose transporter trafficking. Nature 425: 727-733, 2003.

15. Nakata M, Nagasaka S, Kusaka I, Matsuoka H, Ishibashi S and Yada T: Effects of statins on the adipocyte maturation and expression of glucose transporter 4 (SLC2A4): implications in glycaemic control. Diabetologia 49: 1881-1892, 2006.

16. Yamada K, Saito M, Matsuoka H and Inagaki N: A real-time method of imaging glucose uptake in single, living mammalian cells. Nat Protoc 2: 753-762, 2007.

17. Andreozzi F, Laratta E, Sciacqua A, Perticone F and Sesti G: Angiotensin II impairs the insulin signaling pathway promoting production of nitric oxide by inducing phosphorylation of insulin receptor substrate-1 on Ser312 and Ser616 in human umbilical vein endothelial cells. Circ Res 94: 1211-1218, 2004.

18. Birnbaum MJ: Identification of a novel gene encoding an insulinresponsive glucose transporter protein. Cell 57: 305-315, 1989.

19. Charron MJ, Brosius FC III, Alper SL and Lodish HF: A glucose transport protein expressed predominately in insulin-responsive tissues. Proc Natl Acad Sci USA 86: 2535-2539, 1989.

20. James DE, Strube M and Mueckler M: Molecular cloning and characterization of an insulin-regulatable glucose transporter. Nature 338: 83-87, 1989.

21. Kanzaki M and Pessin JE: Insulin-stimulated GLUT4 translocation in adipocytes is dependent upon cortical actin remodeling. J Biol Chem 276: 42436-42444, 2001.

22. Okada T, Kawano Y, Sakakibara T, Hazeki O and Ui M: Essential role of phosphatidylinositol 3-kinase in insulin-induced glucose transport and antilipolysis in rat adipocytes. Studies with a selective inhibitor wortmannin. J Biol Chem 269: 3568-3573, 1994.

23. Corvera S and Czech MP: Direct targets of phosphoinositide 3-kinase products in membrane traffic and signal transduction. Trends Cell Biol 8: 442-446, 1998

24. Khayat ZA, Tong P, Yaworsky K, Bloch RJ and Klip A: Insulininduced actin filament remodeling colocalizes actin with phosphatidylinositol 3-kinase and GLUT4 in L6 myotubes. J Cell Sci 113: 279-290, 2000.

25. Martin SS, Haruta T, Morris AJ, Klippel A, Williams LT and Olefsky JM: Activated phosphatidylinositol 3-kinase is sufficient to mediate actin rearrangement and GLUT4 translocation in 3T3-L1 adipocytes. J Biol Chem 271: 17605-17608, 1996.

26. Andjelković M, Alessi DR, Meier R, et al: Role of translocation in the activation and function of protein kinase B. J Biol Chem 272: 31515-31524, 1997.

27. Alessi DR, Andjelkovic M, Caudwell B, Cron P, Morrice N, Cohen $\mathrm{P}$ and Hemmings BA: Mechanism of activation of protein kinase B by insulin and IGF-1. EMBO J 15: 6541-6551, 1996.

28. Andjelković M, Jakubowicz T, Cron P, Ming XF, Han JW and Hemmings BA: Activation and phosphorylation of a pleckstrin homology domain containing protein kinase (RAC-PK/PKB) promoted by serum and protein phosphatase inhibitors. Proc Natl Acad Sci USA 93: 5699-5704, 1996.

29. Foster LJ, Li D, Randhawa VK and Klip A: Insulin accelerates inter-endosomal GLUT4 traffic via phosphatidylinositol 3-kinase and protein kinase B. J Biol Chem 276: 44212-44221, 2001.

30. Auger KR, Serunian LA, Soltoff SP, Libby P and Cantley LC: PDGF-dependent tyrosine phosphorylation stimulates production of novel polyphosphoinositides in intact cells. Cell 57: 167-175, 1989. 